\title{
Characteristics of soft magnetic composite material under rotating magnetic fluxes
}

\author{
J.J. Zhong, Y.G. Guo*, J.G. Zhu, and Z.W. Lin \\ Faculty of Engineering, University of Technology, Sydney, P.O. Box 123, Broadyway, NSW 2007, Australia
}

* Corresponding author: Tel: +61-2-95147903, Fax: +61-2-95142435, E-mail: youguang@eng.uts.edu.au

\begin{abstract}
This paper reports the measurement of magnetic properties of the soft magnetic composite material SOMALOY ${ }^{\mathrm{TM}} 500$ in a square sample under different patterns of flux density with 2D magnetic excitations. The test system, principle of measurement, magnetic power loss calculation, and methods of correction for misalignment of $\mathrm{H}$ surface sensing coils are presented. The experimental results show that although nominally isotropic, the SOMALOY ${ }^{\mathrm{TM}} 500$ sample exhibits some anisotropy. The results are useful in the design and performance analysis of rotating electrical machines.

(C) 2014 Elsevier B.V. All rights reserved

PACS: 75.50.Gg

Keywords: Soft magnetic composite; Rotating magnetic flux; Magnetization: Misalignment angles; Magnetic anisotropy.
\end{abstract}

\section{Introduction}

Soft magnetic composite (SMC) materials and their application in electromagnetic devices have undergone significant development in the past few years [1]. The basis for the material is the bonded iron powder of high purity and compressibility. The powder particles are bonded with a coating of an organic material, which produces high electrical resistivity. The coated 
powder is then pressed into a solid material using a die and finally heat treated to anneal and cure the bond.

This type of material is in general magnetically isotropic due to its powdered nature and this opens up crucial design benefits for electromagnetic devices. The magnetic circuits can be designed with three-dimensional (3D) flux path and radically different topologies can be exploited to achieve high machine performances because the magnetic flux does not have to be constrained in a plane as that in laminated steels, which are commonly used in electrical machines and transformers [2,3].

With SMC materials being more and more widely used, there is a growing demand for better understanding about the characteristics of the materials, such as the relation between magnetic flux density $\mathbf{B}$ and magnetic field strength $\mathbf{H}$ or $\mathbf{B}-\mathbf{H}$ curve, and magnetic power losses with various kinds of magnetic field excitation. These characteristics are used in the study of magnetization mechanisms, and performance simulation and assessment in design of electromagnetic devices.

In a rotating electrical machine, the magnetic flux density in certain regions follows elliptic or circular orbits. Therefore, the magnetic properties of the core material under rotating fluxes need to be examined and employed in the design and performance simulation of rotating electrical machines. This paper presents the measurement of magnetic properties of the SMC material, SOMALOY ${ }^{\mathrm{TM}} 500$, in a square sample under different patterns of flux density with two-dimensional (2D) magnetic excitations. The test system, principle of measurement, power loss calculation, and correction methods for misalignment of $\mathrm{H}$ surface sensing coils are reported [4]. The experimental results are analyzed and discussed. 


\section{Testing system}

The single sheet tester for square specimen, developed at the University of Technology, Sydney [5], was used for the measurement of the magnetic properties of SMC materials under 2D magnetic excitations. Fig.1 illustrates schematically the testing system.

The 2D magnetic field in the sample is generated by two groups of excitation coils arranged on the $\mathrm{X}$ and $\mathrm{Y}$ axes, respectively. The excitation voltages and currents are supplied via two identical power amplifiers. By controlling the waveforms and by changing the magnitudes and phase angles of the excitation currents on the $\mathrm{X}$ and $\mathrm{Y}$ axes, any combination of one or two dimensional magnetic flux density vectors can be generated. The vector can be an alternating magnetic flux density inclined at a specified angle from the $\mathrm{X}$ or $\mathrm{Y}$ axis, a purely circular or elliptical rotating magnetic flux density, or a rotating magnetic flux density of any specified locus. For the feedback control of the magnetic flux density components on the $\mathrm{X}$ and $\mathrm{Y}$ axes, two specially designed differential amplifiers with low and high pass filters are used. A PC based digital signal processing (DSP) system, AMLAB, is used for both function generation and data acquisition. It is also used for the calculation of the vectors $\mathbf{B}$ and $\mathbf{H}$ and the power losses under alternating or rotational exciting fields.

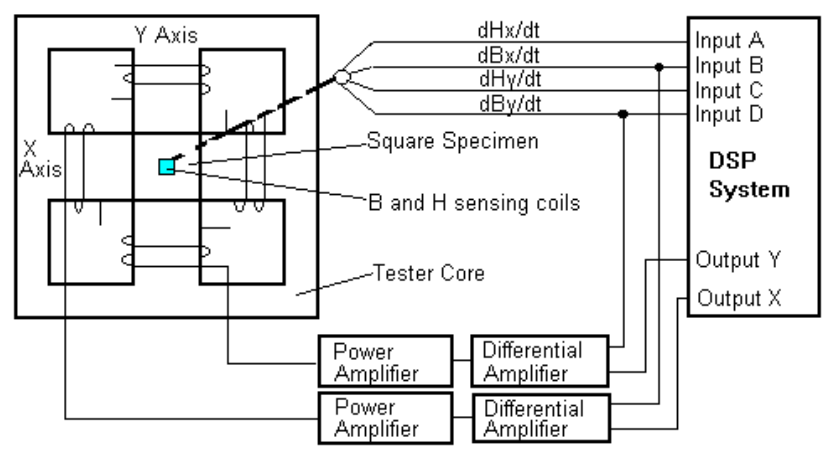

Fig.1 Schematic illustrations of the single sheet testing system 
To determine the total core loss and $\mathbf{B}-\mathbf{H}$ relationship in the sample, the flux density inside the sample and the field strength on the surface should be measured accurately. In this testing, 2D Rogowski-Chattock H-coils are employed for the measurement of the surface field strength. The coils are installed very close to the sample surface to pick up the correct value of the surface field strength. B-coils are used for the measurement of the flux density because of their high accuracy. For measuring the rotational core loss, the field-metric method was employed, featuring high accuracy and great versatility. Moreover, the measured instantaneous $\mathbf{H}$ and $\mathbf{B}$ values can yield additional information, such as various loss contributions, the loci of $\mathbf{H}$ and $\mathbf{B}$ vectors, and harmonics, etc. By the Poynting's theorem, the total core loss $\mathrm{P}_{\mathrm{t}}$ in the samples can be calculated by

$$
\begin{aligned}
P_{t} & =\frac{1}{T \rho_{m}} \int_{0}^{T} \mathbf{H} \bullet \frac{d \mathbf{B}}{d t} d t \\
& =\frac{1}{T \rho_{m}} \int_{0}^{T}\left(H_{x} \frac{d B_{x}}{d t}+H_{y} \frac{d B_{y}}{d t}\right) d t
\end{aligned}
$$

where $\mathrm{T}$ is the time period of one magnetization process, $\rho_{\mathrm{m}}$ the sample mass density, and $H_{\chi}$, $H_{y}, B_{x}$ and $B_{y}$ are the $\mathrm{X}$ and $\mathrm{Y}$ components of $\mathbf{H}$ and $\mathbf{B}$.

\section{Correction of sensing coil misalignment}

If the axes of the $\mathbf{H}$ sensing coils are misaligned with the axes of the excitation coils of the tester, or not perpendicular to each other, the measured values will not be the true components on the $\mathrm{X}$ and $\mathrm{Y}$ axes of the magnetic field. Theoretically, the error caused by this misalignment can be eliminated by a rotation of coordinate axes by using the following 
transformation:

$$
\left(\begin{array}{l}
H_{x} \\
H_{y}
\end{array}\right)=\frac{1}{|C|}\left(\begin{array}{cc}
\cos \alpha_{y} & -\sin \alpha_{x} \\
\sin \alpha_{y} & \cos \alpha_{x}
\end{array}\right)\left(\begin{array}{l}
H_{x}{ }^{\prime} \\
H_{y}{ }^{\prime}
\end{array}\right)
$$

where $\alpha_{x}$ and $\alpha_{y}$ are the angles of misalignment with the $\mathrm{X}$ and $\mathrm{Y}$ axes respectively, $H_{x}$ and $H_{y}$ the true values of the $\mathrm{X}$ and $\mathrm{Y}$ components of the magnetic field strength, $H_{x}{ }^{\prime}$ and $H_{y}{ }^{\prime}$ the measured values of the $\mathrm{X}$ and $\mathrm{Y}$ components of the field strength by the sensing coils, and $|\mathbf{C}|$ $=\cos \alpha_{x} \cos \alpha_{y}+\sin \alpha_{x} \sin \alpha_{y}$.

In practice, however, the discrepancy still exists even though the correction for misalignment has been conducted by the rotating transformation method, as shown in Fig.2. For a clear view of the discrepancy, different scales are used for the $\mathrm{X}$ and $\mathrm{Y}$ axes. The same problem has also puzzled many other researchers [6-9], and the reason is not fully understood yet.

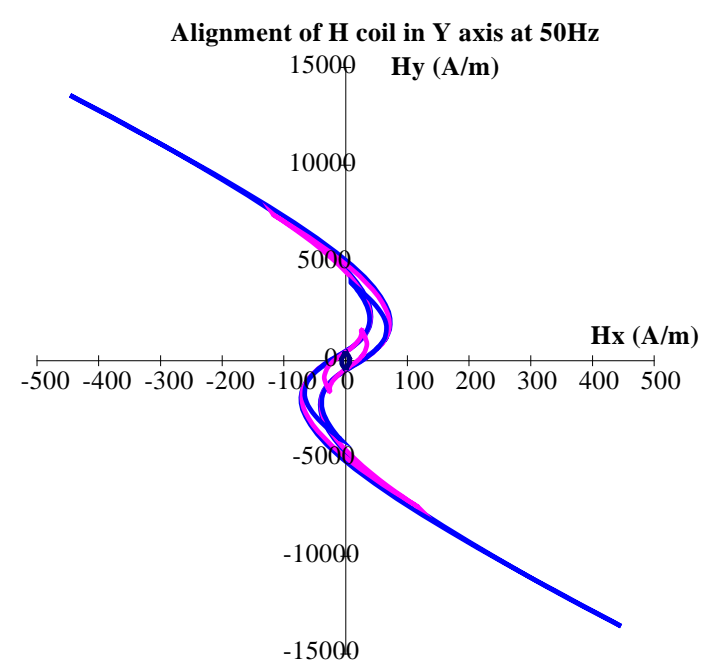

Fig.2 Misalignment of $\mathbf{H}$ due to internal field 
An explanation about the discrepancy is with effect of the internal field, proposed by the authors [4]. Fig.3 illustrates that an alternating field $\mathbf{H}$ acts in $\mathrm{X}$ axis. Due to the effect of magnetization or internal field, the $\mathbf{H}$ vector is dragged away from the $\mathrm{X}$ axis. This leads to an extra or additional misalignment angle. The additional misalignment angle created by $f(\mathbf{M})$ depends on both magnitude of $\mathbf{M}$ and its equilibrium direction.

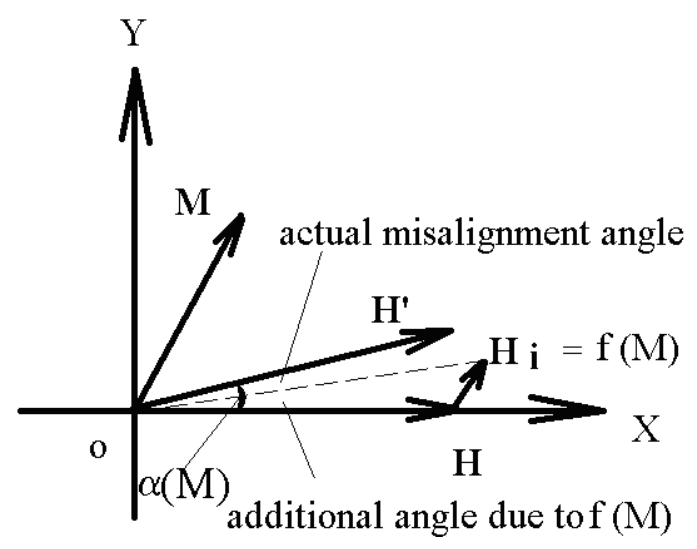

Fig.3 Misalignment angle including actual and additional parts

The measured misalignment angles of $\mathbf{H}$ sensing coils can be simply expressed as

$$
\alpha_{i}(\mathbf{M})=\alpha_{i}+\alpha_{i}(f(\mathbf{M})) \quad(\mathrm{i}=\mathrm{x}, \mathrm{y})
$$

where $\alpha_{i}$ is the actual misalignment angle and $\alpha_{i}(f(\mathbf{M}))$ is the additional misalignment angle due to the effect of internal field, which is related to the magnetization in the material, as illustrated in Fig.3.

Due to the effect of the internal field $\mathbf{H}_{\mathbf{i}}$, it is quite difficult to obtain the correct (actual) misalignment angle, and thus the angular error cannot be corrected completely. This will cause the asymmetry of $\mathbf{H}$ loci and discrepancy of rotational core loss between the two opposite rotating directions. 
As shown in Fig.4, the measured core losses between the clockwise and anti-clockwise directions are different. The difference of losses between the two directions increases with the increment of the flux density B. Even some negative power losses were found. To solve this problem the averaging method is used by averaging the magnetic field strength $\mathbf{H}$ loci and power losses measured with the rotating flux density vectors in opposite rotating directions. The averaging method is derived based on (2) provided that the misalignment angles of sensing coils are small [4].

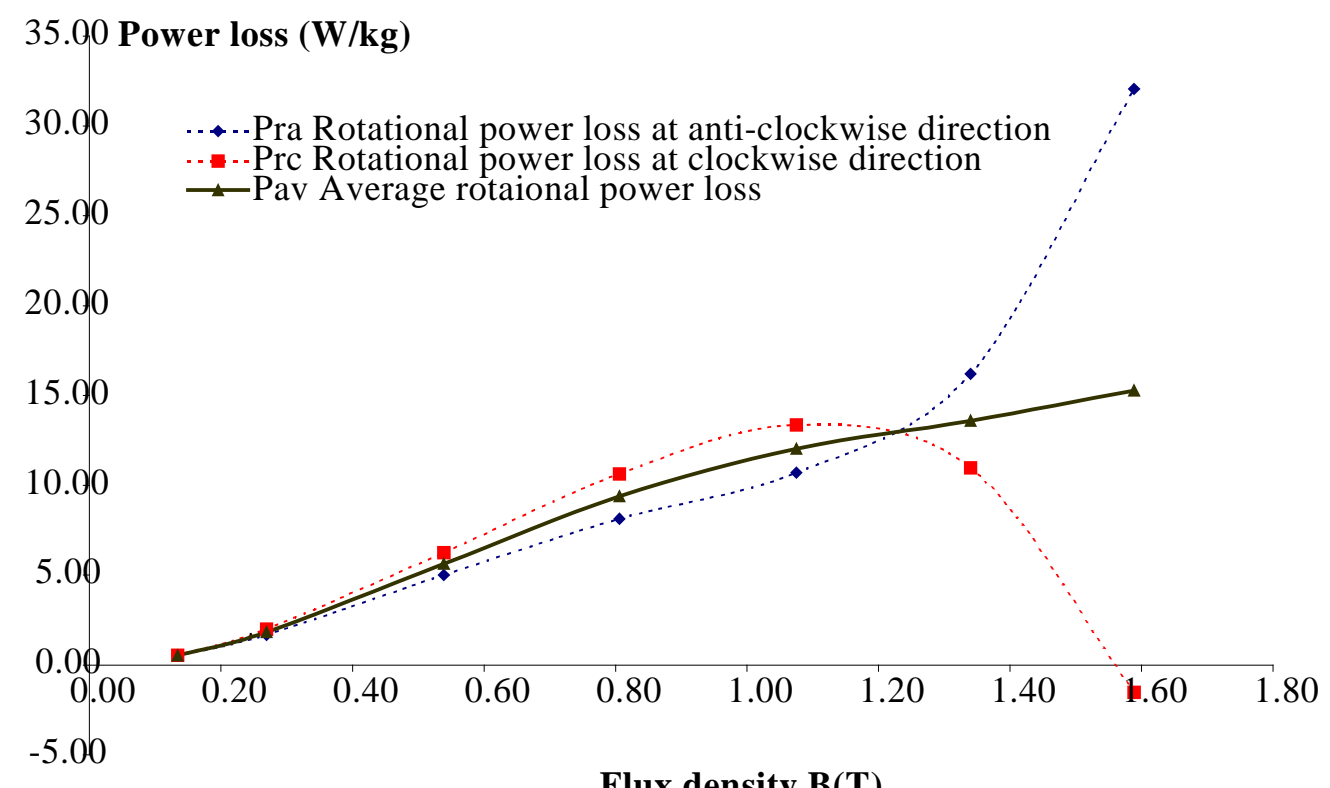

Flux density $B(T)$

Fig.4 Core losses at $50 \mathrm{~Hz}$ with fluxes rotating in clockwise and anti-clockwise directions

\section{Measurement of SMC sample}

A $50 \times 50 \times 1.25 \mathrm{~mm}$ sample of SMC material, SOMALOY ${ }^{\mathrm{TM}} 500$ recently developed by Höganäs AB, Sweden [10], was cut from a cylindrical preform of $80 \mathrm{~mm}$ in diameter and 50 
$\mathrm{mm}$ in height with its normal parallel to the axis of the cylinder. The sample was systematically tested under various circular flux densities and elliptical flux densities as well in the clockwise and anti-clockwise directions at $5 \mathrm{~Hz}, 10 \mathrm{~Hz}, 20 \mathrm{~Hz}, 30 \mathrm{~Hz}, 40 \mathrm{~Hz}, 50 \mathrm{~Hz}$ and $100 \mathrm{~Hz}$ frequencies.

Fig.5 plots the $\mathbf{B}$ and $\mathbf{H}$ loci in the sample with circular rotating flux density vectors at 50Hz. Fig.5(a) illustrates the circular flux density $\mathbf{B}$ and Fig.5(b) shows the rotating field strength $\mathbf{H}$ corresponding to the circular $\mathbf{B}$. The solid lines depict the $\mathbf{H}$ loci after correction by using both rotational coordinate transformation and the averaging method; while the broken lines illustrate the $\mathbf{H}$ loci in the anti-clockwise direction (the loci in the clockwise direction are not shown). It can also be seen that the $\mathbf{B}$ vector is not purely circular when its magnitude is above 1.5T, since it was very difficult to control the flux density $\mathbf{B}$ vector when the specimen was close to saturation (the $\mathbf{H}$ loci corresponding to the rotating $\mathbf{B}$ over $1.5 \mathrm{~T}$ have not been shown). Fig.5(b) also shows that although nominally isotropic, the SOMALOY ${ }^{\mathrm{TM}} 500$ sample measured exhibits some anisotropy.

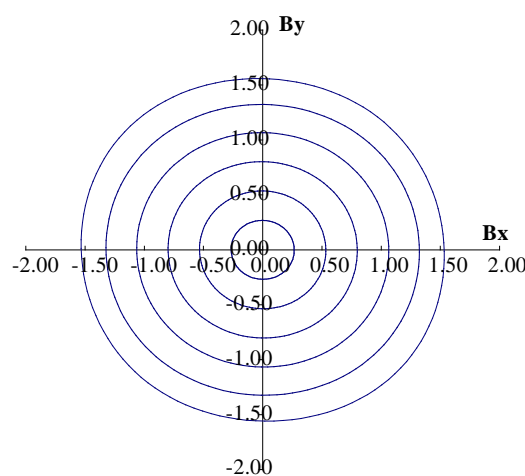

(a)

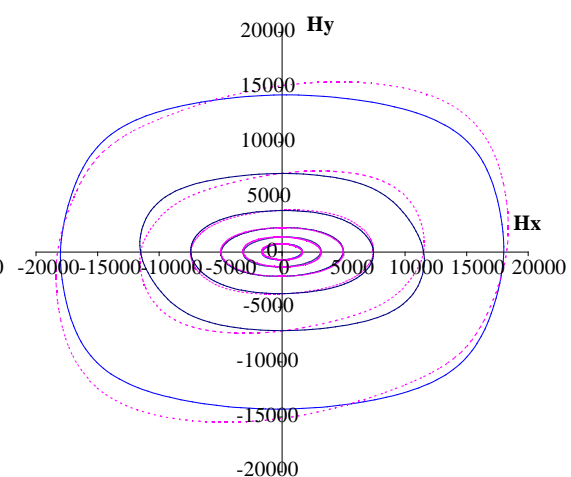

(b)

Fig.5 Loci of (a) $\mathbf{B}$ and (b) $\mathbf{H}$ vectors in sample with circular rotating flux density vector at $50 \mathrm{~Hz}$ 
Fig.6 shows the $\mathbf{B}$ and $\mathbf{H}$ loci of sample versus the axis ratio, which is defined as the ratio between the minor axis and the major axis of an elliptically rotating magnetic flux density at $10 \mathrm{~Hz}$, when the major axis is on the $\mathrm{X}$ and $\mathrm{Y}$ axes respectively. The major axis of the elliptical flux density $\mathbf{B}$ is fixed at 1.5T.

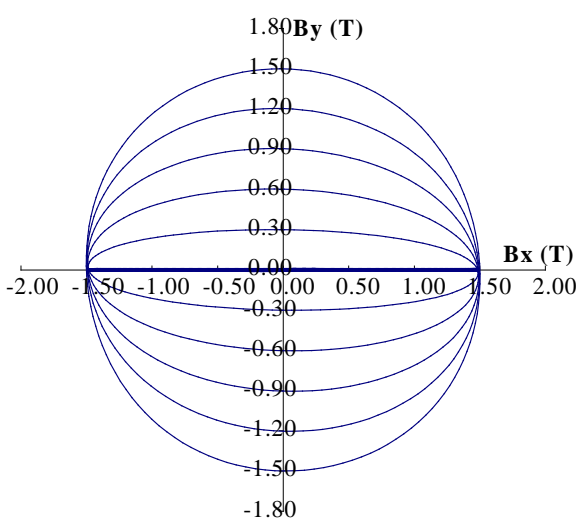

(a)

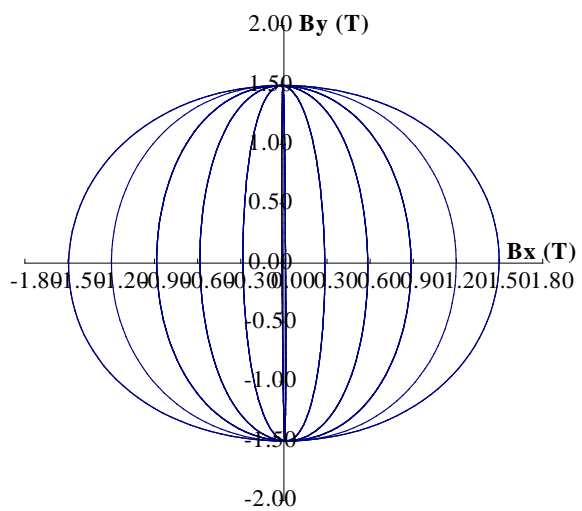

(c)

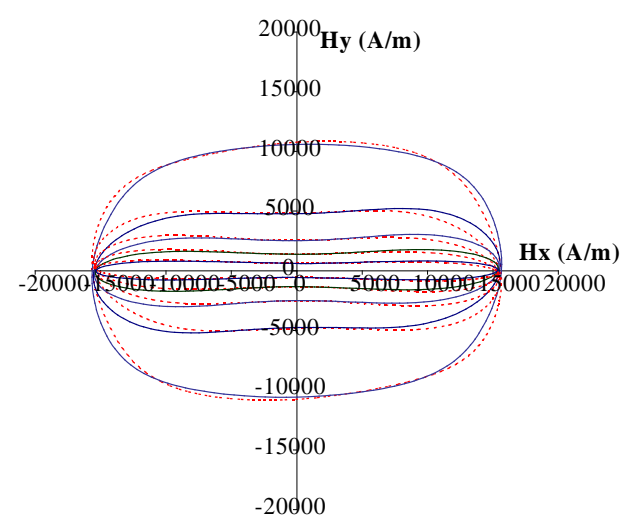

(b)

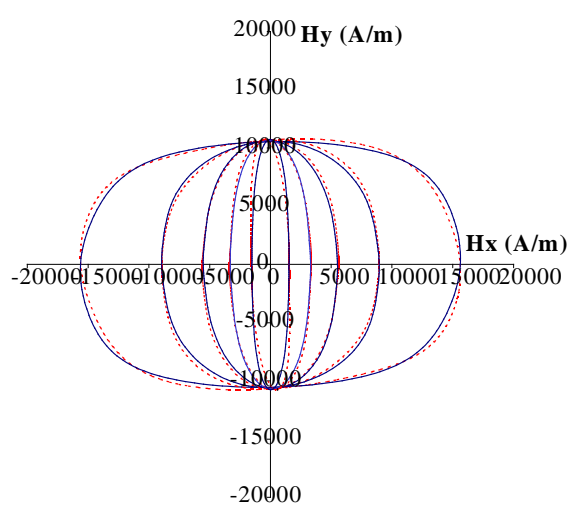

(d)

Fig.6 Loci of $\mathbf{B}$ and $\mathbf{H}$ vectors in sample with elliptical rotating flux density vector at $10 \mathrm{~Hz}$, (a) elliptical $\mathbf{B}$ with major axis on X, and (b) rotating $\mathbf{H}$ corresponding to $\mathbf{B}$, (c) elliptical $\mathbf{B}$ with major axis on $\mathrm{Y}$, and (d) rotating $\mathbf{H}$ corresponding to $\mathbf{B}$. 
The rotational core losses of sample with different circularly rotating flux densities in two directions at $50 \mathrm{~Hz}$ have been shown in Fig.4. It is obvious that the core loss curves between the clockwise and anti-clockwise directions illustrated by broken lines are not coincident, especially in the high flux density area. The possible reason and the solution have been discussed in the last section. The solid lines show a reasonable result by using the average of the data in two directions.

The core losses with 2D circular rotating fluxes at different frequencies are illustrated in Fig.7. The core losses were calculated using both rotational coordinate transformation and the averaging method together. The losses appear to level out when the flux density reaches $1.3 \mathrm{~T}$ but this could be an artifact of the measurement system as high $\mathbf{B}$ measurements are difficult to control.

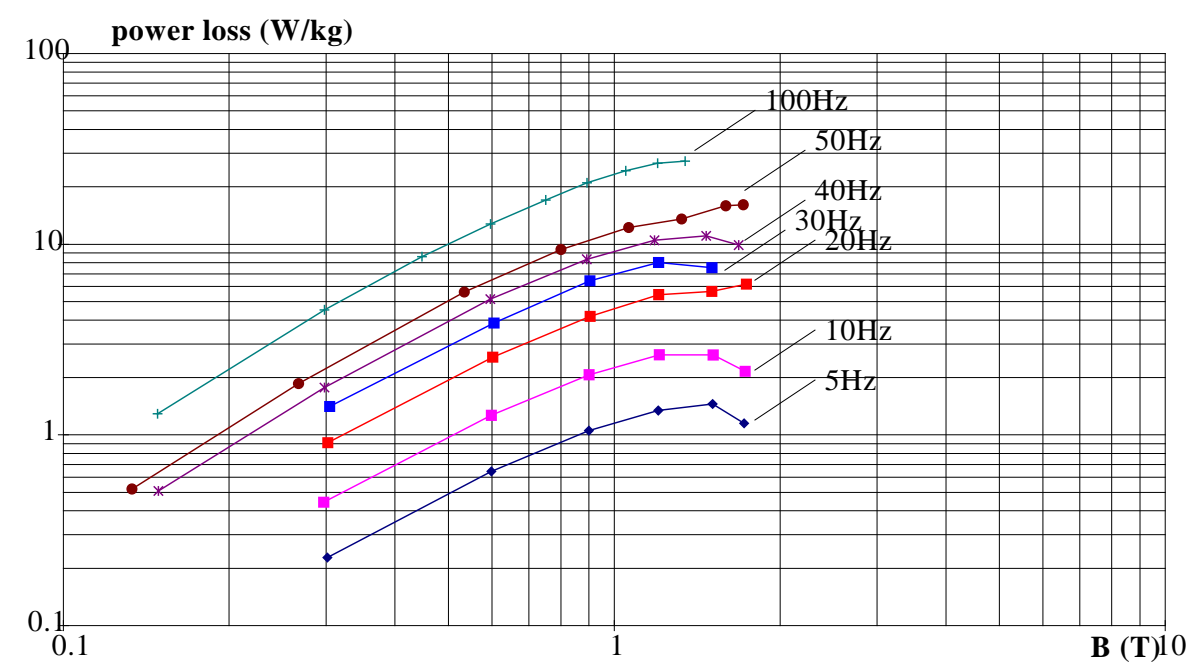

Fig.7 Power loss with 2D circular fluxes at different frequencies 


\section{Conclusion}

The magnetic properties of an SMC material SOMALOY ${ }^{\mathrm{TM}} 500$ have been systematically measured under circular and elliptical rotating magnetic fields of different frequencies. Although nominally isotropic, the SMC sample exhibits some anisotropy. The experimental results will be useful in the design and performance analysis of rotating electrical machines.

\section{Reference:}

[1] "The latest development in soft magnetic composite technology", Reports of Höganäs AB, Sweden, 1997-2005. Available at http://www.hoganas.com, see News then SMC update.

[2] A.G. Jack, "Experience with the use of soft magnetic composites in electrical machines", in Proc. Int. Conf. on Electrical Machines, Istanbul, Turkey, 1998, pp1441-1448

[3] Y.G. Guo, J.G. Zhu, P.A. Watterson, and W. Wu, "Comparative study of 3D flux electrical machines with soft magnetic composite core", IEEE Trans. Industry Applications, Vol.39, Nov./Dec. 2003, pp1696-1703

[4] J.J. Zhong, "Measurement and modelling of magnetic properties of materials with rotating fluxes", PhD thesis, Uni. of Tech., Sydney, Dec. 2002

[5] J.G. Zhu and V.S. Ramsden, "Two dimensional measurement of magnetic field and core loss using square specimen tester", IEEE Trans. Magn, Vol.29, Nov. 1993, pp2995-2997

[6] W. Brix, K.A. Hempel, and F.J. Schulte, "Improved method for the investigation of the rotational magnetization process in electrical steel sheets", IEEE Trans. Magn. Vol.20, Sept. 1984, pp1708-1710 
[7] Y. Alinejad-Beromi, A.J. Moses, and T. Meydan, "New aspects of rotational field and flux measurement in electrical steel", Journal of Magnetism and Magnetic Materials 112 (1992), pp135-138

[8] W. Salz and K.A. Hempel, "Anisotropy of grain-oriented steel sheet under various elliptical field conditions", J. Appl. Phys. Vol.70, Nov. 1991, pp6268-6270

[9] J. Sievert, J. Xu, L. Rahf, M. Enokizono, and H. Ahlers, "Studies on the rotational power loss measurement problem", Anales de Fisica Serie B, Vol.86 ,1990, pp35-37

[10] "Soft magnetic composites from Höganäs metal powders - SOMALOY'M 500", Höganäs Manual, 1997. 